\title{
Sulle Oscillazioni Libere del Mar Ligure (*)
}

\author{
P. Calor - M. C. Spadea
}

Ricevuto il 20 novembre 1960

In un lavoro precedente abbiamo dimostrato la possibilità delle oscillazioni libere del Mar Ligure. Da un calcolo preliminare, basato su di una carta batimetrica insufficientemente precisata (l'isobata piu profonda era limitata a 2000 metri), e risultato un valore per l'uninodale dell'ordine di $2^{\mathrm{l}} 36^{\mathrm{m}}$. In effetti, i valori del periodo dell'uninodale registrati a Genova e Livorno, si aggirano in media sulle $3^{\text {h }}$.

Anche per questo motivo, ottenuta una carta batimetrica del Mar Ligure, recentemente rilevata dall'Istituto Idrografico di Genova nei piu minuti dettagli, ci siamo proposti di ricalcolare il periodo dell'uninodale, estendendo la parte oscillante fino alla linea Cap d'AntibesCapo Calvi, considerata come linea di bocca (fig. 1).

Le sezioni, accresciute di numero, sono state pertanto rideterminate sulla nuova carta batimetrica (fig. 1a). I dati che hanno servito al calcolo sono riportati nella Tabella I.

Considerata una correzione di bocca di 1,5, l'uninodale risulta avere un periodo di $2^{\mathrm{h}} 45^{\mathrm{m}}$ circa. Tenuto conto della grande apertura laterale che il Mar Ligure, considerato come golfo, presenta fra Capo Corso e Piombino, un fattore di correzione di 1,6 sembra piu opportuno. Con tale fattore, il periodo dell'uninodale raggiunge il valore di

$$
T=3^{\mathrm{ln}}, 0 .
$$

E questo il valore medio piu frequentemente osservato.

2 - Ci siamo preoccupati di isolare qualche esempio dell'uninodale del Mar Ligure, registrata a Genova. A tale scopo, occorreva conoscere l'andamento della marea prevista per il porto di Genova. Abbiamo scelto

(*) Questo lavoro è stato eseguito con il contributo del Consiglio Nazionale delle Ricerche. 


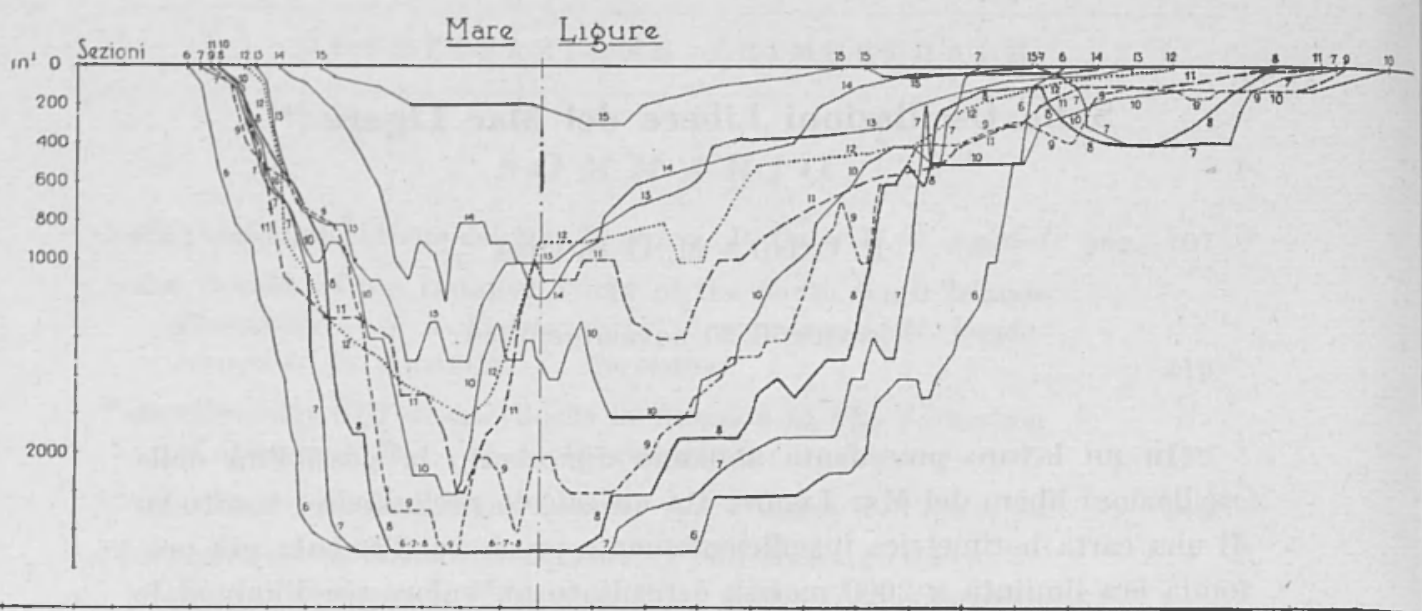

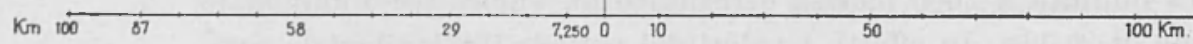

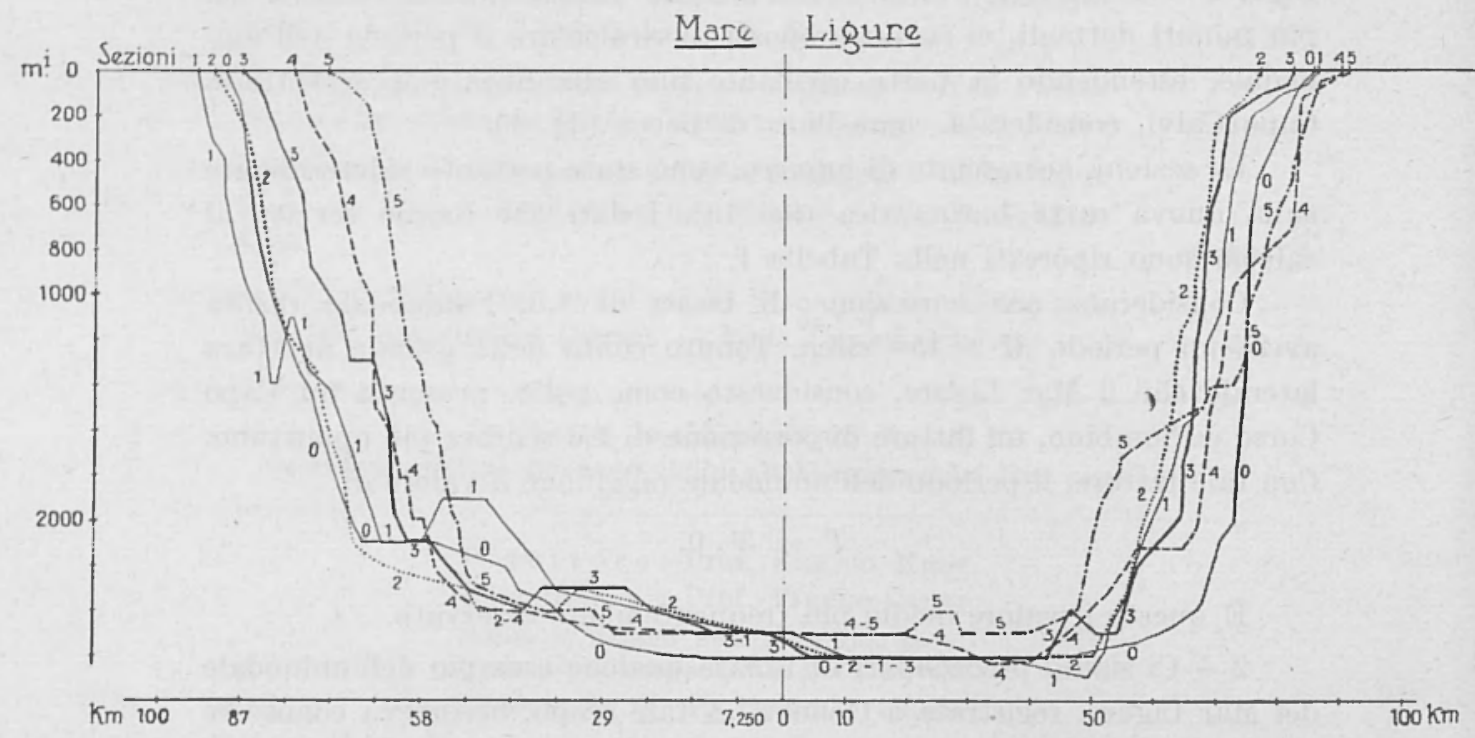

Fig. 1a 


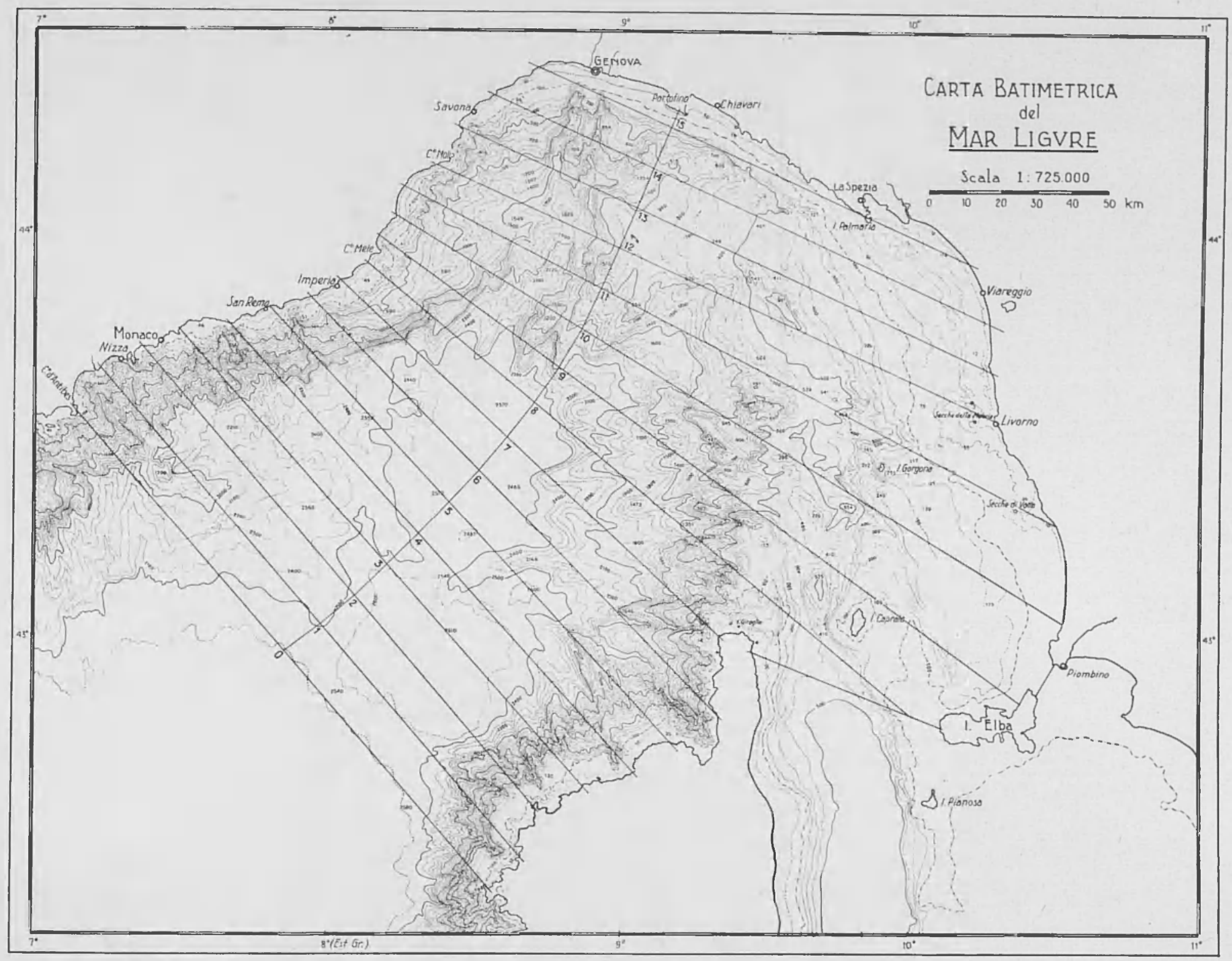

Fig. 1 
per questa indagine le registrazioni ottenute dal mareografo di Genova dal 7 all'11 Giugno 1956 (fig. 2). Per tale periodo ci fu messa cortesemente a disposizione dal Direttore dell'Istituto Idrografico di Genova (che qui nuovamente ringraziamo), la corrispondente marea prevista (fig. 2a). E

Tabella 1

\begin{tabular}{|c|c|c|c|c|c|c|c|}
\hline 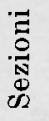 & $\begin{array}{c}\Delta(x)_{v} \\
\mathrm{~m}\end{array}$ & $\begin{array}{c}S(x)_{\nu} \\
10^{6} \mathrm{~m}^{2}\end{array}$ & $\begin{array}{l}\Delta v(x)_{\nu} \\
10^{6} \mathrm{~m}^{2}\end{array}$ & $\begin{array}{l}\xi_{v} \\
\mathrm{~m}\end{array}$ & $\begin{array}{l}\eta_{\nu} \\
\text { in }\end{array}$ & $\begin{array}{c}m_{\nu} \\
10^{10} \mathrm{~m}^{3}\end{array}$ & $T=1^{1 \mathrm{~s}} 50 \mathrm{~m}$ \\
\hline 0 & & 377.49 & 0 & 26491 & 0 & 1.000 & \\
\hline 1 & 12325 & 352.62 & 2349.96 & 28246 & 30.1962 & 0.996 & \\
\hline 2 & $"$ & 346.02 & 2216.45 & 28466 & 62.3644 & 0.985 & \\
\hline 3 & ” & 337.91 & 2103.45 & 28587 & 99.7792 & 0.966 & \\
\hline 4 & $"$ & 340.41 & 2090.84 & 27673 & 132.3172 & 0.942 & \\
\hline 5 & ") & 315.69 & 2037.22 & 28889 & 163.7460 & 0.912 & \\
\hline 6 & $"$ & 319.51 & 2099.77 & 27354 & 196.6538 & 0.874 & \\
\hline 7 & $"$ & 275.89 & 1920.02 & 30193 & 227.7128 & 0.833 & \\
\hline 8 & $"$ & 244.70 & 2394.63 & 31630 & 262.0996 & 0.774 & \\
\hline 9 & ” & 214.92 & 3198.80 & 31826 & 298.0886 & 0.684 & \\
\hline 10 & 》) & 183.39 & 3637.15 & 31027 & 334.3241 & 0.569 & \\
\hline 11 & ") & 158.73 & 3399.58 & 28287 & 369.5736 & 0.449 & \\
\hline 12 & " & 126.71 & 2665.84 & 27306 & 401.7418 & 0.346 & \\
\hline 13 & D & 93.84 & 2081.90 & 27600 & 432.8008 & 0.259 & \\
\hline 14 & " & 68.98 & 1992.55 & 24645 & 464.2296 & 0.170 & \\
\hline \multirow[t]{2}{*}{15} & 8400 & 15.18 & 1787.04 & 55995 & 492.2074 & 0.085 & \\
\hline & & & 702.73 & & 535.6354 & 0.049 & \\
\hline
\end{tabular}

noto che tali determinazioni valgono solo in prima approssimazione, ad ogni modo quello che qui conta essenzialmente l'ordine di grandezza dei valori perseguiti.

Le figg. 3) 4) 5) 6) riportano, in vera grandezza, i risultati delle differenze marea osservata-marea prevista. Tali risultati sono davvero significativi e testimoniano della reale esistenza dell'oscillazione oggetto di questo studio. Abbiamo pertanto ritenuto superfluo estendere l'indagine in questo senso. 

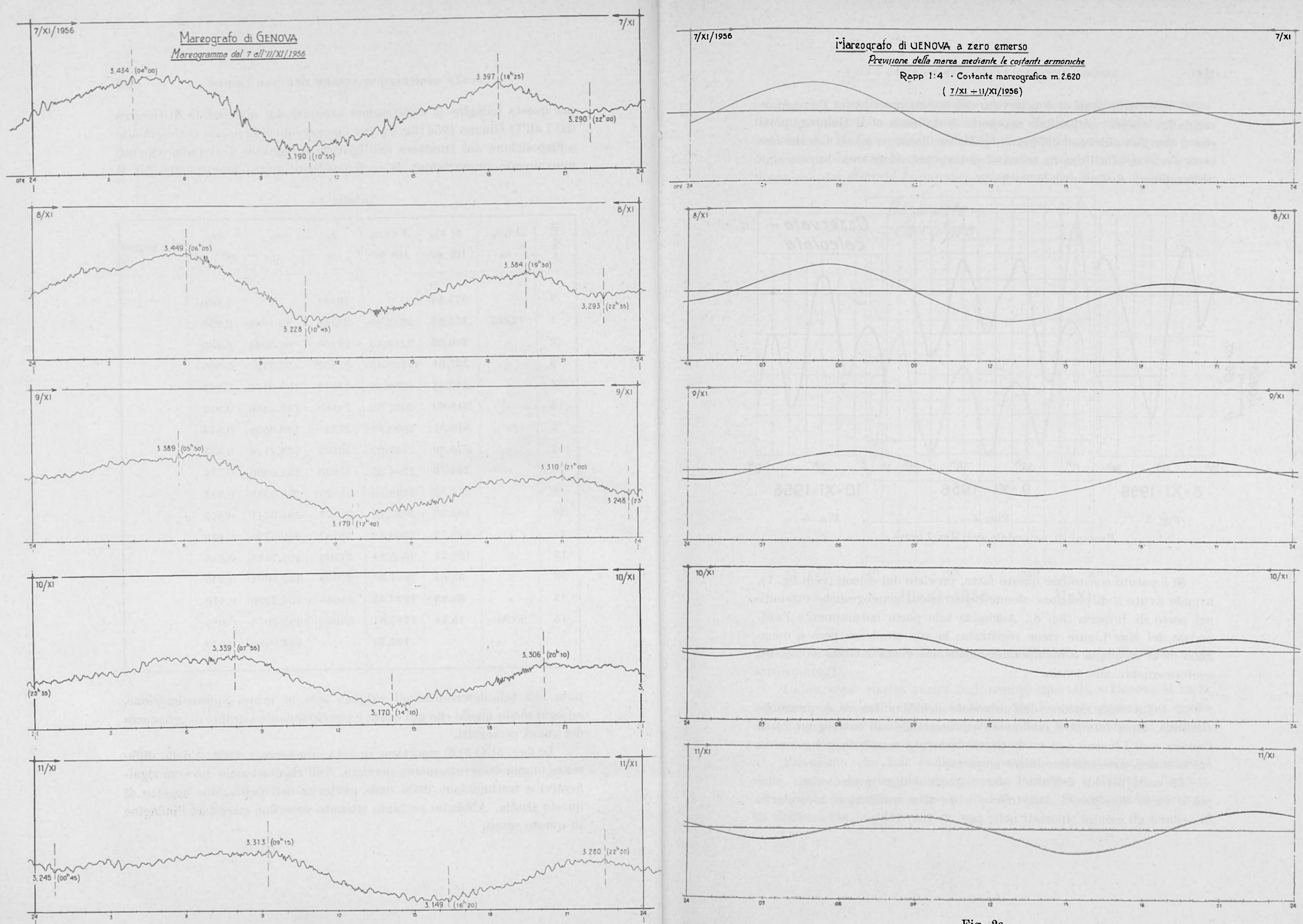

Fig. 2

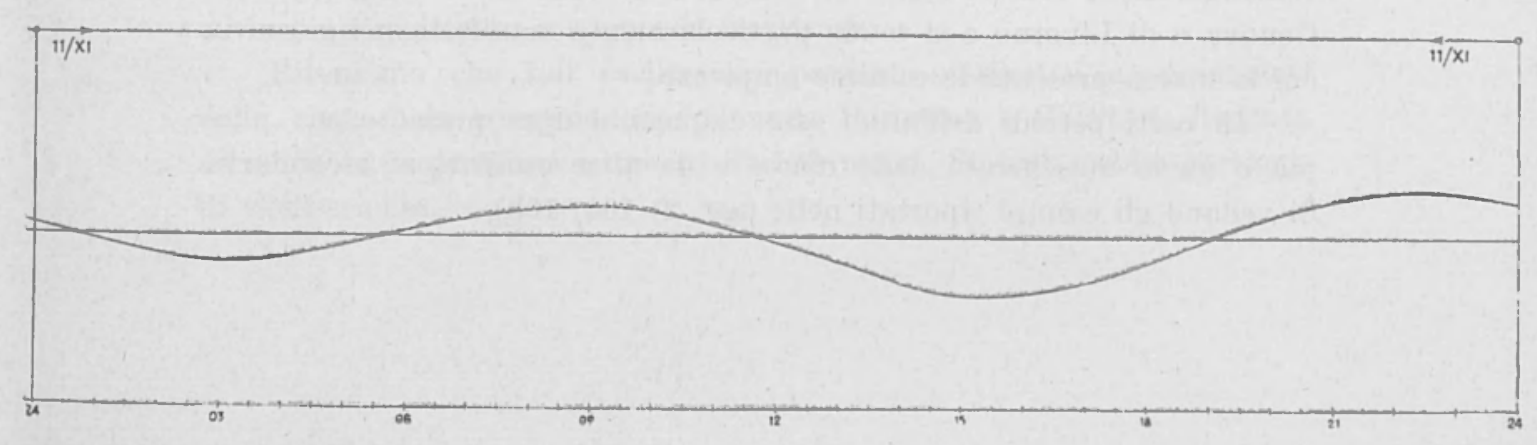

Fig. 2 a 
3 - Mentre, come si e osservato nel lavoro precedente, l'uninodale raggiunge valori comparabili nei porti di Livorno e di Genova, porti che si trovano alla base del grande golfo oscillante, a mano a mano che ci si avvicina alla linea di bocea si poteva prevedere una diminuzione nell'ampiezza dell'uninodale stessa.

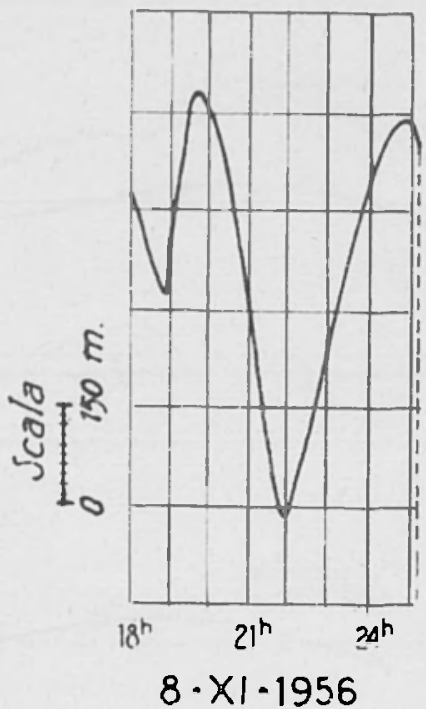

Fig. 3

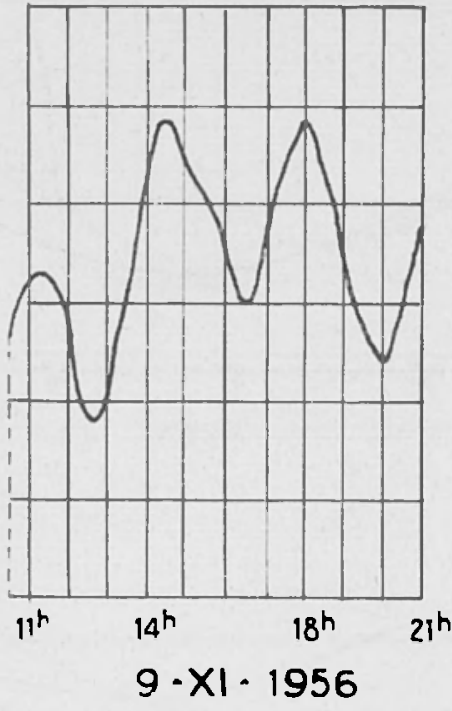

Fig. 4

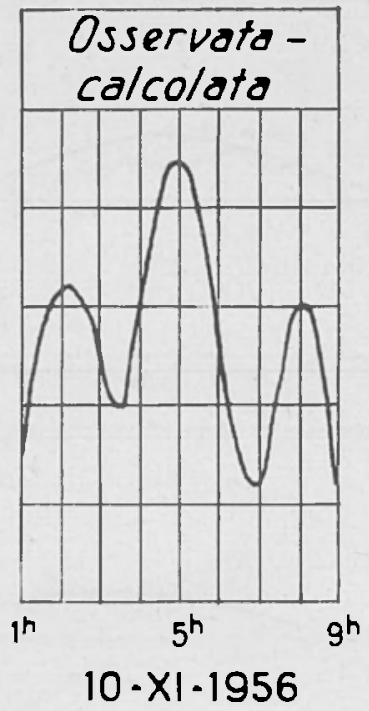

Fig. 5

Esempi di uninodale del Mar Ligure

Si e potuto controllare questo fatto, previsto dai calcoli (vedi fig. T), avendo avuto a disposizione alcune registrazioni mareografiche ottenute nel porto di Imperia (fig. 8). Anche in tale porto naturalmente l'uninodale del Mar Ligure viene registrata; la sua ampiezza pero è meno della meta di quella contemporaneamente osservata a Genova (fig. 9), conformemente alla teoria.

4 - La registrazione dell'uninodale del Mar Ligure e pressoche continua. Essa infatti e facilmente rintracciabile sui mareogrammi di Genova e di Livorno e si rende particolarmente manifesta nei giorni in cui la marea presenta le minime ampiezze.

In certi periodi dell'anno essa e quasi sempre presente, sia pure piu o meno mascherata dalle maree e da altre oscillazioni secondarie. Si vedano gli esempi riportati nelle figg. 2) 10a) 10b). 
5 - Per quanto si riferisce alle cause di questa oscillazione riteniamo fondamentale quella associata al transito di disturbi microbarici, provenienti dal Mediterraneo Occidentale. I massimi spostamenti alla base del Golfo si dovrebbero pertanto riscontrare quando la velocita di propagazione dei disturbi barografici provenienti dal largo e diretti verso

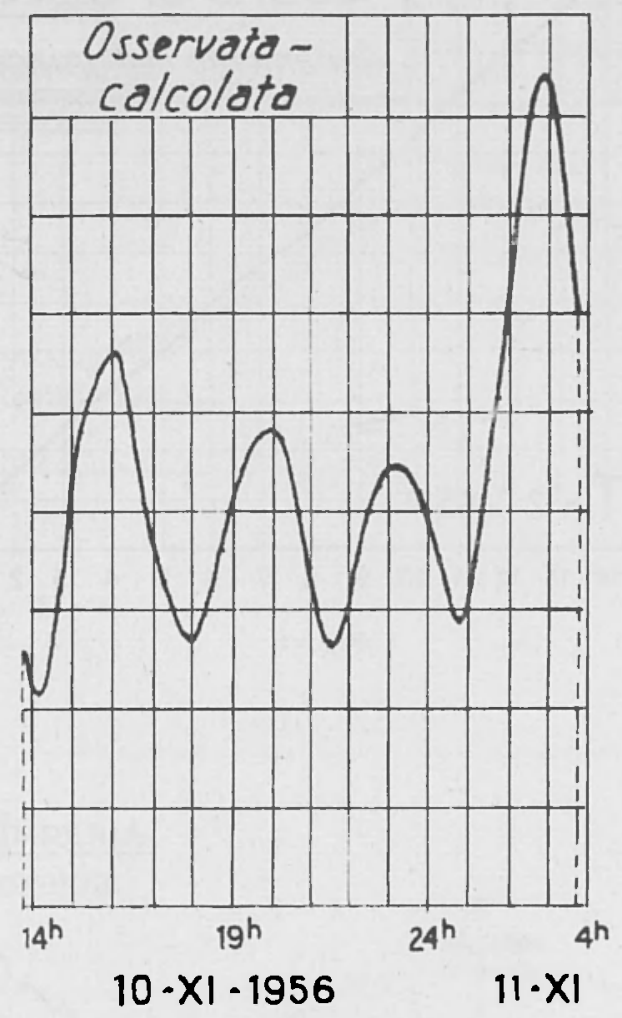

Fig. 6

la base del golfo stesso, è dell'ordine di quella delle onde libere del mare sottostante $\left({ }^{2}\right)$.

Infine, come risulta anche dagli esempi riportati, a Genova si ha la registrazione di oscillazioni secondarie, fra le quali prevalgono quelle aventi un periodo di $25^{\mathrm{m}}$ circa (fig. 2). Sovente, hanno ampiezze sensibili e vengono registrate con grande frequenza.

Riteniamo che tali oscillazioni possano attribuirsi a movimenti della vasta baia comprendente Genova Portofino e Chiavari, limitata da un'isobata prossima a quella di 100 metri. Si tratterebbe pertanto di shelf-seiches. 


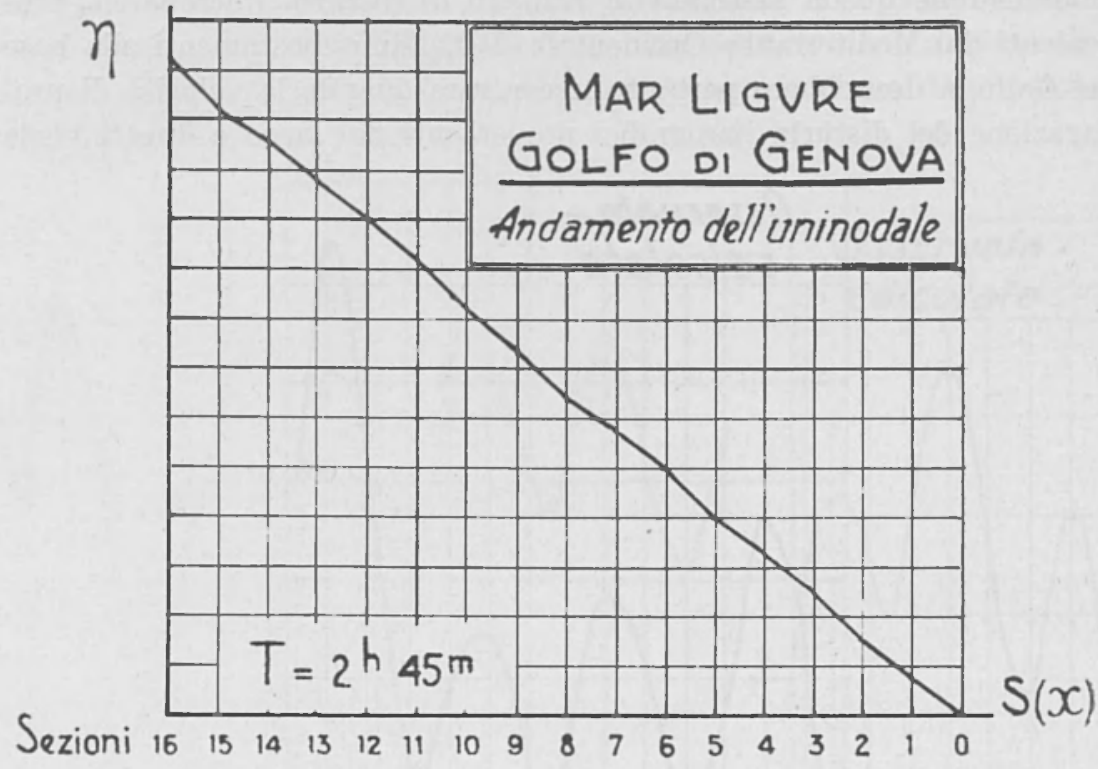

Fig. 7

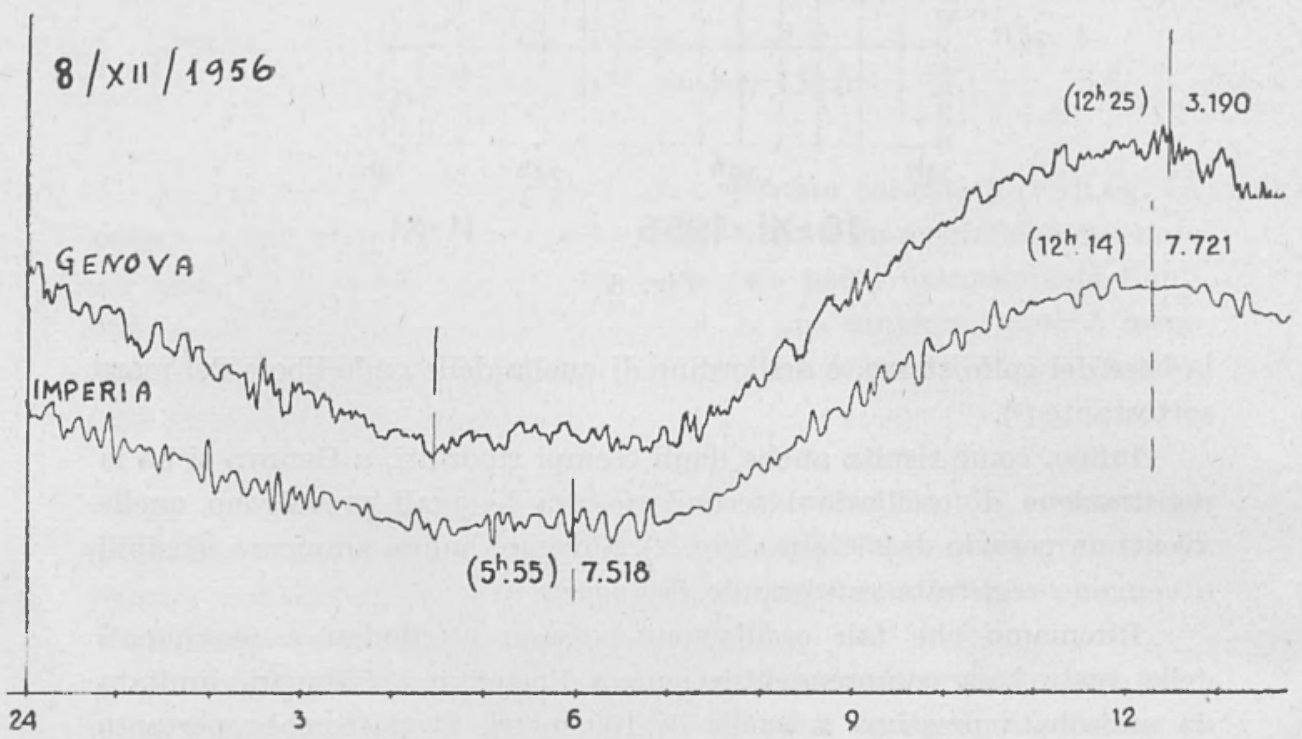

Fig. 8 

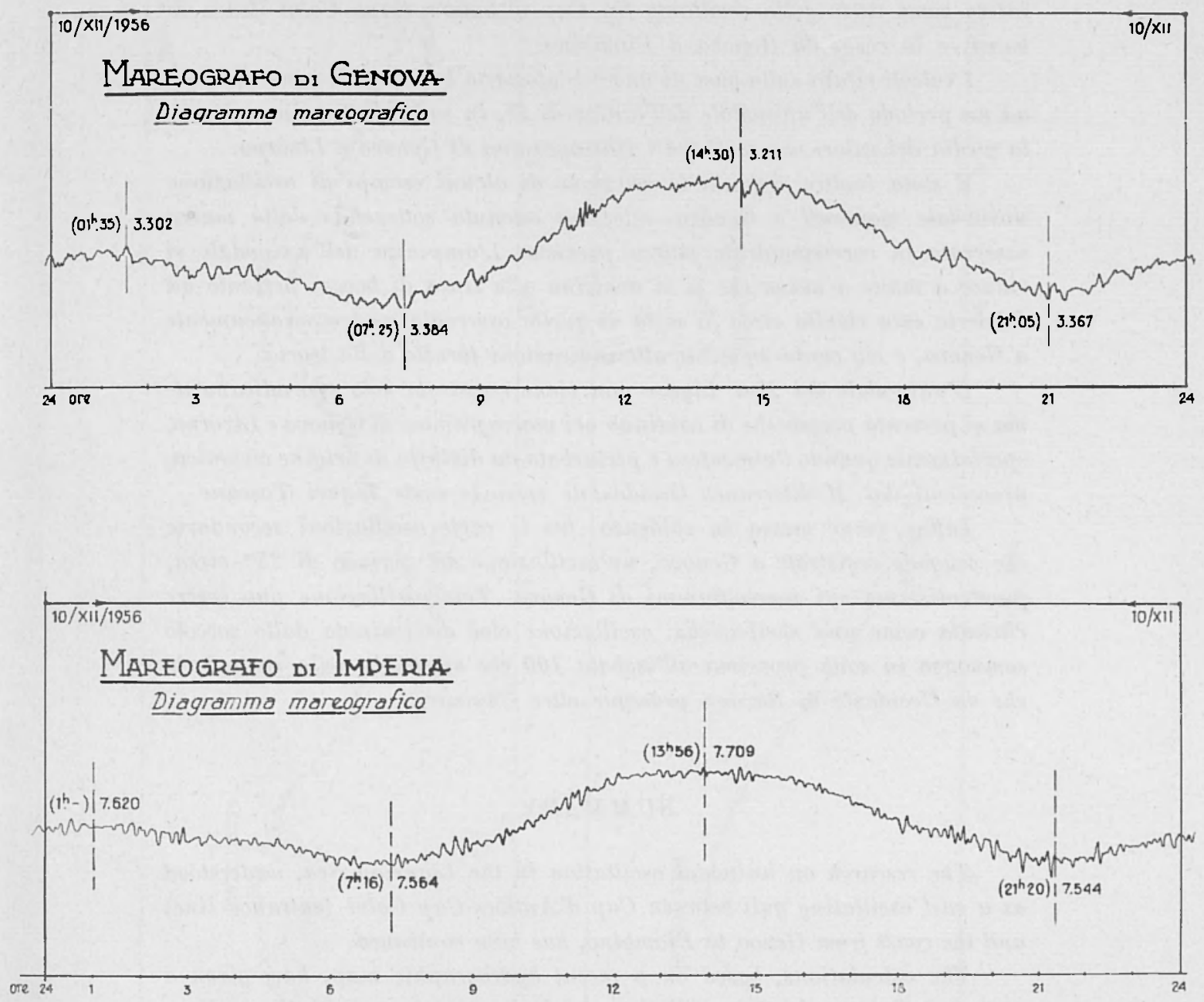

Fig. 9 


\section{RIASSUNTO}

E' stata proseguita la ricerca sull'oscillazione uninodale del Mar Ligure, inteso come vasto golfo oscillante fra Cap d'Antibes-Capo Calvi (linea di bocsa) e la costa da Genova a Piombino.

I calcoli rifatti sulla base di una recente carta batimetrica hanno portato ad un periodo dell'uninodale dell'ordine $d i 3^{h}$, in soddisfacente accordo con la media dei valori osservati fra $i$ mareogrammi di Genova e Livorno.

E stata inoltre eseguita la selezione di alcuni esempi di oscillazione uninodale registrati a Genova, selezione ottenuta sottraendo dallu marea osservata la corrispondente marea prevista. L'ampiezza dell'uninodale si riduce a mano a mano che ci si avvicina alla linea di bocca: pertanto ad Imperia essa risulta circa la meta di quella osservata contemporaneamente a G'ienova, e ciò conformemente alle indicazioni fornite dalla teoria.

L'uninodale del Mar Ligure non viene registrata solo sporadicamente ma si presenta pressocche di continuo nei mareogrammi di Genova e Livorno, specialmente quando l'atmosfera e perturbata da disturbi di origine ciclonica, procedenti dal Mediterraneo Occidentale verso le coste Liguri Toscane.

Infine, viene messa in evidenza, fra le varie oscillazioni secondarie che venyono registrate a Genova, un'oscillazione del periodo di $25^{\mathrm{m}}$ circa, frequentissima sui mareogrammi di Genova. Tale oscillazione puo essere ritenuta come una shelf-seiche: oscillazione cioe determinata dallo zoccolo sommerso in zona prossima all'isobata 100 che si stende nella vasta baia che da Occidente di Savona prosegue oltre Chiavari.

\section{$S U M M A R Y$}

The research on uninodal oscillation in the Ligurian Sea, understood as a vast oscillating gulf between Cap d'Antibes-Cap Calvi (entrance line) and the coast from Genoa to Piombino, has been continued.

The calculations, based on a recent hydrographic map, have given a period of $3^{h}$ for uninodal oscillation, fairly in accordance with the medium values, observed on the mareograms in Genoa and Livorno.

Besides, some examples of uninodal oscillations, as registered in Genoa, have been selected. The selection was made by subtracting from the actually observed tide, the corresponding foreseen tide. The ampleness of uninodal oscillations is less and less pronounced, the nearer one comes to the entrance line; consequently it is only about one half of the oscillations 


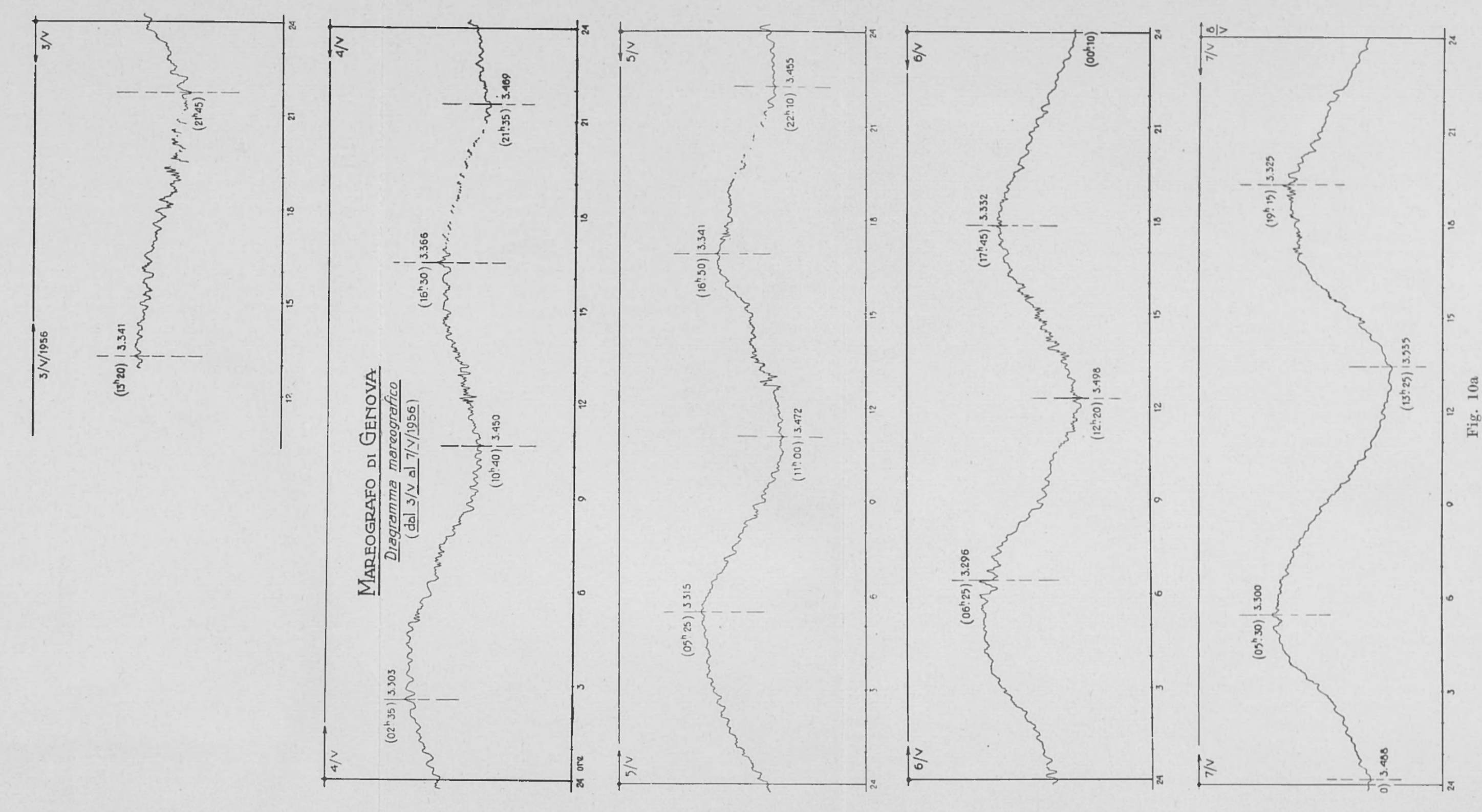




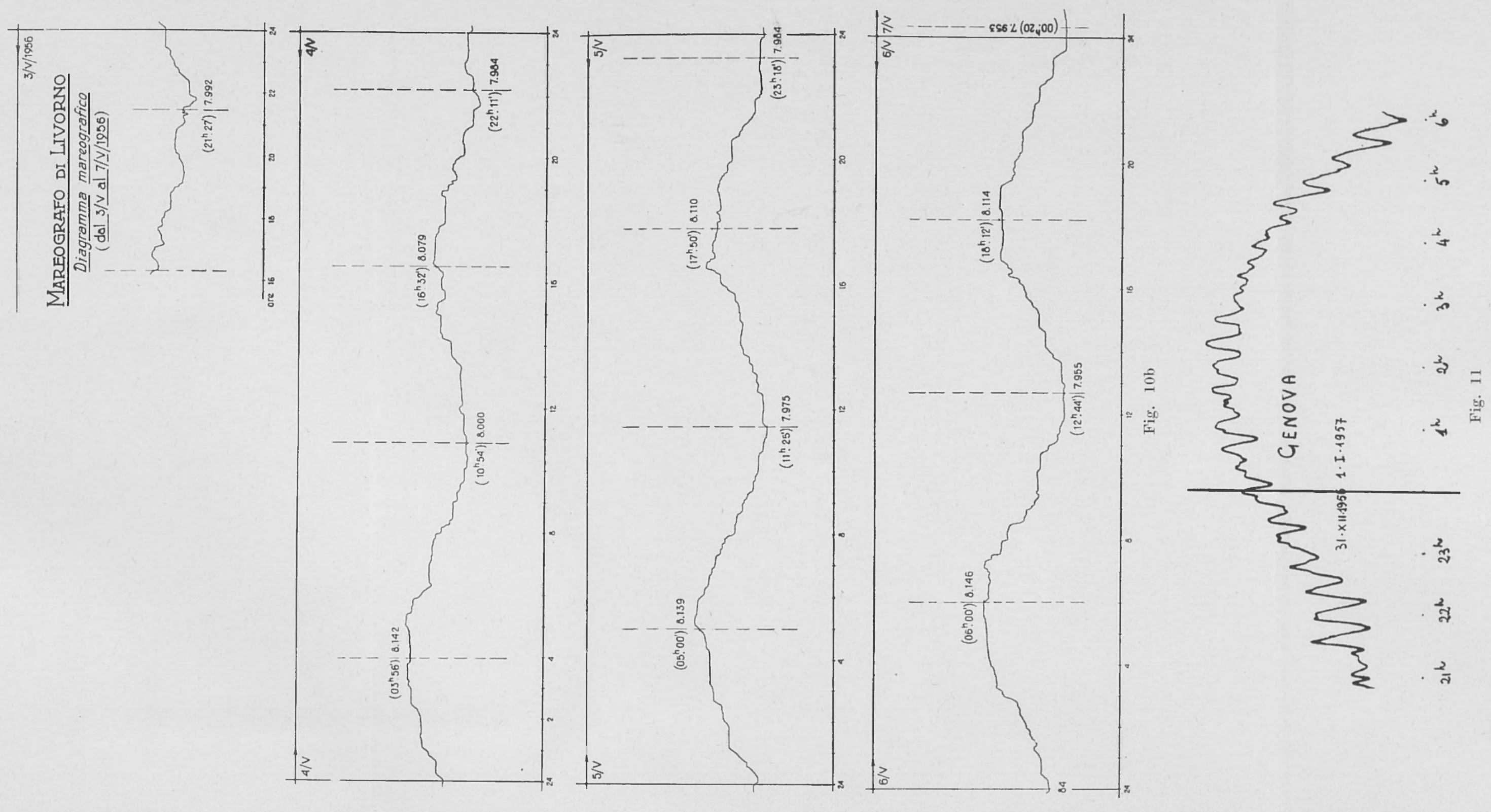


which are observed, at the same time, in Genoa. And this according to the values worked out in theory.

Uninodal oscillation in the Ligurian Sea is not registered sporadically, but almost continually in the mareograms of Genoa and Livorno, particularly at times of perturbations of cyclonic origin, which approach the Ligurian and Tuscan coasts from the Eastern Mediterranean.

Finally, among the various secondary oscillations, which are registered in Genoa, there is to be mentioned specially one oscillation of about $25^{\mathrm{m}}$ period, which is very frequent on the mareograms of Genoa. This oscillation may be considered a "shelf-seiche", that is an oscillation caused by the submerged mass, situated near isobath 100, in the vast bay beginning west from Savona and ending beyond Chiavari.

\section{BIBLIOGRAFIA}

(') Calor P., Le sesse del Lago di Garda, Parte II, "Annali di Geofisica ", $1,2,1948$.

$\left({ }^{2}\right)$ - Sesse dell'Alto Adriatico con particolare riguardo al Golfo di Trieste, R. Com. Tass. Italiano, 1938. 